\title{
A importância da Fisioterapia Bucomaxilofacial para a Cirurgia Oral Menor
}

\author{
The importance of Bucomaxillofacial Physiotherapy for Minor Oral Surgery \\ La importancia de la Fisioterapia Bucomaxilofacial para la Cirugía Oral Menor
}

Recebido: 09/07/2021 | Revisado: 15/07/2021 | Aceito: 16/07/2021 | Publicado: 25/07/2021

Emmanuele Figueiredo Marcião

ORCID: https://orcid.org/0000-0003-4011-8073

Faculdade Pitágoras de Santarém, Brail

E-mail: emmanuelemarciao@gmail.com

Gabriel Lucas Moura Batista

ORCID: https://orcid.org/0000-0002-0304-4055

Universidade da Amazônia, Brazil

E-mail: gabrielmourabiomed@gmail.com

Jamilly Maria dos Santos Rabelo ORCID: https://orcid.org/0000-0001-6713-6293 Universidade da Amazônia, Brazil

E-mail: jamillymaria19@gmail.com

Sadriane Lima Fernandes

ORCID: https://orcid.org/0000-0001-5689-0813

União das Escolas Superiores de Rondônia, Brazil

E-mail: sadrifernandes@gmail.com

Sara Juliana de Almeida Branches

ORCID: https://orcid.org/0000-0001-8763-8370

Universidade Federal do Oeste do Pará, Brazil E-mail: sarabranches@gmail.com

Mônica Natividade Melgarejo Pedroso

ORCID: https://orcid.org/0000-0002-8158-7709 Universidade Luterana do Brasil, Brazil E-mail: monimelp@hotmail.com

Geremias da Silva

ORCID: https://orcid.org/0000-0002-3331-7907 Faculdade Pitágoras de Santarém, Brazil E-mail: geremias.stm.silva@gmail.com

\begin{abstract}
Resumo
Cirurgia oral menor é uma subespecialidade da cirurgia bucomaxilofacial, sendo responsável por procedimentos cirúrgicos relacionados a ossos, dentes, gengivas e bochechas. Envolve o diagnóstico, tratamento e acompanhamento de extrações de dentes, pequenas lesões e traumatismos na região da boca, maxilar e regiões próxima (Pallota,2020). O diagnóstico é realizado pelo cirurgião dentista e é eminentemente clínico por meio de exame físico intra e extra- oral. $\mathrm{O}$ tratamento da dor pós-operatória deve ser feito de modo regular e atender às necessidades individuais de cada paciente, respeitando seu limiar de dor, bem como outros aspectos inerentes ao paciente (Rocha et al.,2007). O objetivo deste artigo é verificar através de um levantamento da literatura a incidência da cirurgia oral menor nos últimos quinze anos e a importância da fisioterapia. Trata-se de um estudo de revisão bibliográfica por meio de uma busca de manuscritos de caráter científico publicado nos últimos quinze anos (2005 a 2021), realizado a partir da consulta de material de leitura de qualidade acadêmica. Ao observar os resultados obtidos, o presente artigo possibilitou a análise da fisioterapia bucomaxilofacial, a qual tem como principal objetivo a reabilitação das estruturas faciais do paciente com técnicas, exercícios e condutas especializadas para cada quadro.
\end{abstract}

Palavras-chave: Fisioterapia bucomaxilofacial; Cirurgia oral menor; Intervenção cirúrgica; Dor pós-operatória.

\begin{abstract}
Minor oral surgery is a subspecialty of maxillofacial surgery, being responsible for surgical procedures related to bones, teeth gums and cheeks. It involves the diagnosis, treatment and monitoring of tooth extractions, minor injuries and trauma in the mouth, jaw and surrounding regions. (Pallota, 2020). Diagnosis is performed by the dental surgeon and is eminently clinical through intra and extra- oral physical examination. Postoperative pain treatment must be done and meet the individual needs of each patient, respecting their pain threshold, as well as other aspects inherent to the patient (Rocha et al, 2007). The objective of this article is to verify, through a surgery of the literature, the incidence of minor oral sugery in the last fifteen years and the last fifteen years ( 2005 a 2021), carried out by consulting academic quality reading material. By observing the results obtained, this article enable the analysis of maxillofacial physioterapy, which has as its main objective the rehabilitation of the patient's facial structures with techniques, exercises and specialized procedures for each condition.
\end{abstract}

Keywords: Maxillofacial physioterapy; Minor oral surgery; Surgical intervention; Postoperative pain. 


\section{Resumen}

La cirugía oral menor es una subespecialidad de la cirugía maxilofacial, siendo responsable de los procedimientos quirúrgicos relacionados con huesos, dientes, encías y mejillas. Implica el diagnóstico, tratamiento y seguimiento de extracciones dentales, lesiones menores y traumatismos en la boca, la mandíbula y las regiones circundantes. (Pallotta,2020). El diagnóstico lo realiza el cirujano dentista y es eminentemente clínico a través de la exploración física intra y extraoral. El tratamiento del dolor postoperatorio debe realizarse con regularidad y satisfacer las necesidades individuales de cada paciente, respetando su umbral de dolor, así como otros aspectos inherentes al paciente (Rocha,2007). El objetivo de este artículo es verificar, a través de un relevamiento de la literatura, la incidencia de cirugías bucales menores en los últimos quince años y la importancia de la fisioterapia. Se trata de un estudio de revisión de la literatura la búsqueda de manuscritos científicos publicados en los últimos quince años (2005 a 2021), realizada mediante la consulta de material de lectura de calidad académica. Al observar los resultados obtenidos, este artículo permitió el análisis de la fisioterapia maxilofacial, que tiene como principal objetivo la rehabilitación de las estructuras faciales del paciente con técnicas, ejercicios y procedimientos especializados para cada condición.

Palabras clave: Fisioterapia maxilofacial; Cirugía oral menor; Intervención quirúrgica; Dolor posoperatorio.

\section{Introdução}

A cirurgia maxilofacial é realizada por cirurgiões maxilofaciais especializados no tratamento de traumas e anormalidades do complexo maxilo-mandibular, dor orofacial, enxertos ósseos e implantes dentários na região oral. Geralmente são seletivos, entretanto os riscos devem ser minimizados. Deste modo, essas cirurgias podem afetar a respiração, os músculos e as articulações, assim como a cirurgia ortognática e a cirurgia da articulação temporomandibular (ATM). Portanto, a presença de profissionais como fonoaudiólogos e fisioterapeutas é de grande valia para o acompanhamento e tratamento desses pacientes.

A fisioterapia atua junto à equipe de cirurgia bucomaxilofacial desde o pré-operatório, com uma avaliação minuciosa e completa que deve conter informações sobre doenças sistêmicas prévias, função respiratória, função da ATM, sensibilidade orofacial e identificação dos fatores de risco para complicações pós-operatórias. No pós-operatório imediato a presença do fisioterapeuta é de extrema importância principalmente nas cirurgias realizadas em âmbito hospitalar, com o paciente sob anestesia geral. Isto devido ao risco de complicações respiratórias relacionadas com a intubação (muitas vezes são pacientes com via aérea difícil) e a ventilação mecânica, a manipulação intensa de tecidos na face e comunicação com as vias aéreas já que é uma cirurgia realizada por dentro da cavidade oral, o que aumenta os riscos de broncoaspiração. (Valente,2017)

Assim, no pós operatório imediato, faz-se necessária uma avaliação respiratória detalhada e, quando necessárias, condutas afim de evitar e tratar complicações pulmonares e relacionadas ao edema de face e pescoço. Além da questão pulmonar e de vias aéreas, o fisioterapeuta pode atuar dentro da equipe

bucomaxilofacial de forma a tratar o edema advindo dessas cirurgias, minimizando assim o desconforto e a dor referida por esses pacientes. Quanto à motricidade orofacial, cabe ao fisioterapeuta o tratamento de possíveis complicações traumáticas e cirúrgicas como a paralisia facial, auxiliando no retorno às funções normais e protegendo o globo ocular. Além disso, a orientação do fisioterapeuta sobre exercícios de mímica facial facilita muito a recuperação dos movimentos orofaciais. No pós operatório tardio, a atenção volta-se para a ATM, para garantir função dentro dos padrões de normalidade e evitar aderências articulares. (Valente,2017)

A assistência envolve desde o suporte inicial em pré-operatório a à reabilitação total do mesmo. Uchôa et al (2020). Percebe-se que a atuação da fisioterapia é fundamental, visto que os pacientes que realizam tais procedimentos cirúrgicos necessitam desde o pré-operatório até o pós-operatório, uma maioratenção, dessa forma, objetiva-se descrever a importância da fisioterapia bucomaxilofacial.

Para que o paciente tenha condições de receber alta, alguns parâmetros devem ser avaliados, como o estado de consciência, os sinais vitais, presença de diurese espontânea e a alimentação por via oral. Pacientes com fixação maxilomandibular podem ter dificuldade na ingestão de medicamentos na forma sólida (comprimidos, drágeas, cápsulas), 
requerendo assim que estes sejam prescritos em solução ou em gotas. (Carvalho et al, 2010)

É de fundamental importância que toda a equipe responsável pelo atendimento ao trauma em ambiente hospitalar, incluindo o cirurgião bucomaxilofacial, tenha amplo domínio sobre a logística que seestende desde a admissão, evolução e alta hospitalar do paciente. (Carvalho et al, 2010)

A presença do fisioterapeuta na equipe multiprofissional visa reduzir riscos, prevenir ou tratar complicações e restaurar a função de forma mais rápida, segura e eficaz. Porém, ainda é um campo pouco explorado e muito fragmentado, e poucos profissionais entendem não só a função e fisiologia das estruturas envolvidas, mas também as operações realizadas. Portanto, a exploração do conhecimento nesta área garante a diferenciação da formação do fisioterapeuta. De acordo com a cirurgia, para melhor atenderesses pacientes e evitar distrações nos cuidados, os profissionais devem compreender o tipo de cirurgia e a técnica cirúrgica, bem como a adaptabilidade das técnicas e recursos fisioterapêuticos para a região da face, pescoço e boca conforme cirurgia realizada.

O principal objetivo desta pesquisa é fazer um levantamento de dados da fisioterapia bucomaxilofacial e a reabilitação da estrutura facial do paciente. Essa sendo a etapa básica para que o paciente se recupere de maneira efetiva. A fisioterapia bucomaxilofacial no pós-operatório trás

procedimentos adequados às mais diversas situações para que os resultados sejam positivos do tratamentoe que sejam de recuperação rápida. Portanto, o fisioterapeuta tem o compromisso de controlar o edema que pode ocorrer após a cirurgia, para que a função facial e os músculos afetados voltem ao normal mais rapidamente, principalmente no caso de pequenas cirurgias orais.

\section{Metodologia}

A análise dos estudos demográficos e epidemiológicos mostra que em termos relativos, nos últimos quinze anos (de 2005 a 2020), apenas em 2010 que houve mais necessidade do fisioterapeuta no pós-operatório de cirurgia oral menor, o que nos levou a fazer este levantamento de dados, afinal é uma área pouco explorada.

Há uma escassez de estudos na literatura que destaquem a fisioterapia bucomaxilofacial para o tratamento préoperatório e operatório, apenas dados que corroboram com os achados nos estudos de Cunha, et al (2018); Bridi, Ribeiro, et al (2010 a 2013) e Silva, et al (2011) no tratamento pós-operatório com uso de fármacos e exercícios fisioterapêuticos.

De acordo com Lucked (2013), para esta pesquisa, deve- se ter foco e observação nas abordagens qualitativas de pesquisa, as quais são determinadas por propósitos específicos do estudo, que por sua vez são analisadas em um quadro geral feito pelo próprio pesquisador. Com esses propósitos em mente, o observador inicia a coleta de dados buscando sempre manter uma perspectiva de totalidade, sem se desviar demasiado de seus focos de interesse. Para isso, é particularmente útil que oriente a sua observação em torno de alguns aspectos. Visando este suporte metodológico, a pesquisa possui natureza quantitativa, trazendo um levantamento de dados comprobatórios relevantes a pesquisa em si.

Considerando a importância da metodologia da pesquisa no desenvolvimento do artigo, optou-se por uma análise quantitativa que proporcionará estreita relação com a pesquisa. Trata-se de um estudo de revisão bibliográfica por meio de uma busca de manuscritos de caráter cientifico publicado nos últimos quinze anos (de 2005 a 2020), realizado a partir da consulta de material da leitura de qualidade acadêmico em : Scientifc Electronic Library Online (SciELO), Medline, PubMed, Ministério da Saúde, Secretária do Estado de Saúde do Distrito Federal, Protocolos da SES/DF, RR Medicina e Odontologia. Procurou-se identificar artigos científicos que se encaixassem nos seguintes critérios de inclusão: estudos epidemiológicos relacionados à cirurgia oral menor. Onde foram selecionados artigos que eram compostos por análises de prontuários e estatísticas. Todos os dados presentes na tabela são do Brasil, idioma português e ano de publicação 2010 a 2018.

Através das informações coletadas nos anos de 2010 a 2018 foi possível identificar e classificar em tabela de acordo 
com: fonte, local, amostra, a cirurgia oral menor realizada e resultados com a fisioterapia. A apresentação dos dados quantitativos é por tabela, a qual os programas utilizados foram Word e Excel, com o propósito de mostrar informações confirmatórias para análise da importância da fisioterapia bucomaxilofacial para uma melhor recuperação após a cirurgia oral menor.

\section{Resultados e Discussão}

Foram encontrados três estudos relevantes sobre cirurgia oral menor. Esses dados estão presentes na Tabela 1, a seguir:

Tabela 1. Artigos selecionados para a pesquisa.

\begin{tabular}{|c|c|c|c|c|c|}
\hline Fonte & Local & Amostra & $\begin{array}{l}\text { Cirurgia oral } \\
\text { menor }\end{array}$ & Recurso & $\begin{array}{l}\text { Resultado com } \\
\text { fisioterapia }\end{array}$ \\
\hline $\begin{array}{c}\text { Cunha, L. S., } \\
\text { Braga, L. L. } \\
\text { A., Lima, C. } \\
\text { C., Araújo, L. } \\
\text { O., Mota, I. B. } \\
\text { de O., \& } \\
\text { Souza, G. R. } \\
(2018) \\
\end{array}$ & $\begin{array}{c}\text { Patos de } \\
\text { Minas } \\
\text { (MG) }\end{array}$ & $\begin{array}{c}46 \\
\text { Pessoas }\end{array}$ & $\begin{array}{l}\text { Extração de } \\
\text { siso }\end{array}$ & $\begin{array}{l}\text { Redução de dor e edema } \\
\text { com anti-inflamatórios }\end{array}$ & $\begin{array}{c}\text { Apresentou } \\
\text { melhoras após } 7 \\
\text { dias com } \\
\text { fármacos e } \\
\text { repouso e } \\
\text { exercícios de } \\
\text { fisioterapia. }\end{array}$ \\
\hline $\begin{array}{l}\text { Bridi, M. } \\
\text { das P., } \\
\text { Ribeiro, } \\
\text { et al } \\
(2010 \text { a } \\
2013)\end{array}$ & $\begin{array}{l}\text { Grande } \\
\text { Vitória } \\
(\mathrm{ES})\end{array}$ & $\begin{array}{c}1286 \\
\text { Pessoas }\end{array}$ & $\begin{array}{l}\text { Cirurgias } \\
\text { pré- protéticas }\end{array}$ & Reabsorção óssea & $\begin{array}{c}\text { Apresentou } \\
\text { melhoras após } \\
\text { realizar cirurgia e } \\
\text { com exercícios } \\
\text { obteve melhor } \\
\text { desenvol- } \\
\text { vimento } \\
\end{array}$ \\
\hline $\begin{array}{l}\text { Silva, T. F. A, } \\
\text { Souza, R. } \\
\text { B.,Rocha,R. D., } \\
\text { Araújo, F. A. C., } \\
\text { Morais, H. H. A. } \\
\text { (2011) }\end{array}$ & $\begin{array}{l}\text { Rio } \\
\text { Grande } \\
\text { do Norte } \\
(\mathrm{RN})\end{array}$ & $\begin{array}{c}75 \\
\text { pessoas }\end{array}$ & $\begin{array}{l}\text { Biopsia na } \\
\text { cavidade oral }\end{array}$ & $\begin{array}{l}\text { retirada de uma } \\
\text { amostra de tecido vivo } \\
\text { na região bucal para } \\
\text { análise }\end{array}$ & $\begin{array}{l}\text { Após o resultado do } \\
\text { diagnóstico, Foram } \\
\text { divididas em biópsias } \\
\text { excisionais e incionais } \\
\text { (não houve } \\
\text { procedimento } \\
\text { fisioterapêutico) }\end{array}$ \\
\hline
\end{tabular}

Fonte: Autores (2021).

Ao avaliar os prontuários de pacientes submetidos às cirurgias em Pato de Minas, no período de fevereiro a setembro de 2018, foram analisados prontuários referentes às indicações cirúrgicas de extração de siso, extração de dentes anteriores, cirurgias periodontais e, ainda, patologias associadas. De acordo com a análise de 46 prontuários (100\%), $45,66 \%$ destes foramextrações de siso. Foram extraídos 37 sisos, destes 32,43\% foram do elemento 18, 37,83\% do elemento 28, 16,21\% do elemento 38 e 13,51\% do elemento 48. Do total, 4,34\% são de gengivoplastia, referente a cirurgia periodontal. (Cunha, et al 2018).

Diante dos resultados apresentados, pode-se sugerir que a maior prevalência de cirurgias foi de extrações dentárias, sendo 45,66\% de extrações de siso. (Cunha, et al 2018). Na análise das cirurgias pré- protéticas, de um total de 1.286 prontuários, 77 pacientes foram submetidos a esse tipo de cirurgia (cirurgias pré-protéticas), correspondendo a 5,98\% dos pacientes, ou seja, das 77 cirurgias realizadas no período do estudo, 62 foram em mulheres e 15 em homens. A idade variou entre 30 e 85 anos. (Bridi et al, 2010 a 2013). Em relação à procedência dos pacientes, 71 cirurgias (92,2\%) foram realizadas em pacientes moradores da Grande Vitória, região metropolitana, e 06 cirurgias(7,8\%), em pacientes procedentes do interior do estado do Espírito Santo.( Bridi et al, 2010 a 2013). Ao observar os resultados obtidos com Biopsia na cavidade oral, desse total, 75 pacientes apresentaram alguma lesão no Complexo Buco-maxilo-facial, dos quais 30 lesões 
tiveram indicações para realização de biópsias para posterior exame histopatológico.

O total de pacientes acometidos com lesões corresponde a 18,38\% do total. Do total de pacientes submetidos a biópsias, 10 foram do sexo masculino e 20 do sexo feminino; quanto à cor da pele, 27 eram Leucodermas, e 03, Melanodermas; quanto à faixa etária, 42 anos como média, sendo a faixa de 41 a 60 anos a mais comum (13 casos). Foram realizados 30 (trinta) envios de pedidos de exame histopatológico ao Departamento de Patologia Oral da Universidade Federal do Rio Grande do Norte, o que possibilitou a chegar a um diagnóstico mais preciso para os pacientes. ( Silva, et al, 2011).

\section{Considerações Finais}

No presente estudo verificou-se que as pesquisas epidemiológicas relacionadas a cirurgia oral menor, sendo que dentre os artigos selecionados, a cirurgia bucal mais realizada foi a cirurgia pré-protética, a qual possui como finalidade corrigir alterações ósseas e fibromucosas, inserções musculares ou remoção de lesões das quais são possíveis causas de uma má adaptação de uma prótese total ou parcial. Em seguida, a cirurgia de biopsia bucal, a qual inclui amostras de tecido vivo do corpo humano para análise, sendo necessária quando há confirmação de lesão na cavidade oral, e como terceira cirurgia, a extração do siso, a qual é necessária quando não há espaço para os terceiros molares.

Nesse sentido a fisioterapia bucomaxilofacial tem como principal objetivo a reabilitação das estruturas faciais do paciente com técnicas, exercícios e condutas especializadas para cada quadro, o fisioterapeuta atua no controle dos edemas que podem surgir na fase do pós-operatório. Por consequência, as funções faciais e os músculos afetados, voltarão com mais rapidez ao normal. É um passo fundamental no pós-operatório para a recuperação do paciente ser de fato efetiva e considerada um sucesso.

Como todos os setores da sociedade, a cirurgia oral menor está passando por mudanças e se adaptando a novas condutas na área da saúde, as quais foram possíveis notar a presença de fisioterapeutas para a reabilitação no pós-operatório. Futuramente, espera-se que os fisioterapeutas possam atuar no pré-operatório com orientações até no operatório e não apenas no pós-operatório para reabilitar. Sabe-se que o fisioterapeuta irá preparar o paciente com exercícios, alongamentos e movimentos que farão parte do processo de reabilitação. Ao passar um tempo com um fisioterapeuta, ajudará o paciente a se acostumar com esses movimentos e não será tão difícil após sua cirurgia. O tempo de recuperação será mais eficiente do que a fisioterapia apenas no pós-operatório. Em virtude do cenário atual, com escassez de estudos na literatura que destaquem a fisioterapia bucomaxilofacial para o tratamento pré-operatório e operatório, faz-se necessários o desenvolvimento de trabalhos futuros referente ao tema em busca de uma melhor qualidade de vida aos pacientes que necessitarem realizar uma cirurgia oral menor e explorar este campo da fisioterapia.

\section{Referências}

Bassanezi, B. S. B., Oliveira Filho, A. G. (2006). Analgesia Pós- Operatória. Rev Col Bras Cir. 33(2), 116-22. https://www.scielo.br/scielo.php?pid=S0100$69912006000200012 \&$ script $=$ sci_abstract $\&$ tlng=pt

Bridi, M. P., Ribeiro, E. T., Bertollo, R. (2016). Prevalência de cirurgias pré-protéticas em pacientes atendidos na disciplina de cirurgia bucomaxilofacial II da UFES. Rev Brasileira de Pesquisa em Saúde. 17(1), 73-80. https://periodicos.ufes.br/article/view/12452

Carvalho, M. F. D., Herrero, R. K. R., Moreira, D. R., Urbano, E. S., \& Reher, P. (2010). Princípios de atendimento hospitalar em cirurgia buco-maxilo-facial. Revista de Cirurgia e Traumatologia Buco-maxilo-facial, 10(4), 79-84. http://revodonto.bvsalud.org/scielo.php?script=sci_arttext\&pid=S180852102010000400012

Cunha, L. S., Braga, L. L. A., Lima, C. C., Araújo, L. O., Mota, I. B. de O., \& Souza, G. R. (2018). Análise quantitativa dos pacientes submetidos à clínica cirúrgica na policlínica da Faculdade Patos de Minas. Psicologia E Saúde Em Debate, 4(Suppl1), 46-46. http://psicodebate.dpgpsifpm.com.br/index.php/periodico/article/view/392 
Fattah, C. M. R. S., Aranega, A. M., Leal, C. R., Martinho, J. \& Costa, A. R. (2005). Controle da dor pós-operatória em cirurgia bucal: revisão de literatura. Rev Odontol Araçatuba. http://revodonto.bvsalud.org/scielo.php?script=sci_nlinks\&ref=445627\&pid=S1808-5210201000040001000010\&lng=es

Figueiredo, C. M. B. F., Minari, I. S., Bonardi, J. P., Ponzoni, D., Brandini, D. A., \& Bassi, A. P. F. (2020). Epidemiological profile of pediatric maxillofacial trauma: a 20-year retrospective study of patients treated by a university graduate service in Araçatuba, Brazil. Research, Society and Development, 9(8), e989986722. https://doi.org/10.33448/rsd-v9i8.6722

Lima, M. C. de , Toledo Neto, J. L. , Giordani ,A. T. , Bravo, D. S., Katakura, E. A. L. B. , Marzola ,C. (2017). Contribuição para a criação de protocolo de enfermagem em pós-operatório imediato de cirurgia bucomaxilofacial. Brazilian Journal of Surgery and Clinical Research - BJSCR.18,.133-137. https://www.researchgate.net/publication/318042233

Ludke, M. \& Andre, M. E. D. A. (2013). Pesquisas em educação: uma abordagem qualitativa. E.P.U. https://edisciplinas.usp.br/pluginfile.php/4091392/mod_resource/content/1/Lud_And_cap3.pdf

Pallota, R. (2020). Cirurgia oral menor: o que é e quais seus procedimentos. RR Medicina. https://rrmedicina.com.br/cirurgia-oral-menor-o-que-e-e-quais-seusprocedimentos

Rocha, A. P. C., Kraychete, D. C., Lemonica, L. (2007). Dor: Aspectos atuais da Sensibilização Periférica e Central. Revista Brasileira de Anestesiologia, 57(1), 94-105. https://www.scielo.br/j/rba/a/6MtJvgjXNzZqzRgY4x9WXGB/?format=pdf\&lang=pt

Santos, D. M., et al (2021). Cirurgia e Traumatologia Bucomaxilofacial em tempos de pandemia COVID-19: Gestão, riscos e perspectivas. Research, Society and Development , 10 (4), e53310414477. https://doi.org/10.33448/rsd-v10i4.14477

Santos, H. L. F. dos, Barreto, J. O., Pereira, T. L. da S., Bastos, C. F. de B., Medeiros, M. S., Freire, J. C. P., \& Ribeiro, E. D. (2020). Factors influencing fear and anxiety in patients undergoing minor oral surgery and dental implants: a literature review. Research, Society and Development, 9(9), e544996657. https://doi.org/10.33448/rsd-v9i9.6657

Silva, R. C., Souza, F. A., Garcia, J. et al (2015). Atendimento cirúrgico para pacientes vítimas de trauma bucomaxilofacial em nível hospitalar e ambulatorial. UNESP. https://repositorio.unesp.br/handle/11449/142588

Soller, I. C. D. S., Poletti, N. A. A., Beccaria, L. M., Squizatto, R. H., Almeida, D. B. D., \& Matta, P. R. A. (2016). Perfil epidemiológico de pacientes com traumatismos faciais atendidos em emergência hospitalar. Revista Mineira de Enfermagem. http://www.reme.org.br/artigo/detalhes/1069

Uchôa, I. S., Freitas, F. A. P. S., Magalhães, M. do A. V., Nascimento, S. T. T., \& Costa, G. R. (2020). Importance of nursing care for patients with oral maxillofacial trauma. Research, Society and Development, 9(11), e1449119580. https://doi.org/10.33448/rsd-v9i11.9580

Valente, A. C. B. (2017). A presença do fisioterapeuta na equipe de cirurgia bucomaxilofacial. Curso de abordagem fisioterapêutica nas cirurgias bucomaxilofaciais. Sede da Physio Cursos São Paulo. https://www.physiocursossp. com.br/post/fisioterapia-na-cirurgia-bucomaxilo 